\title{
Comparison the Effect of Two Periods of Aerobic Training and One Period of Detraining on the Quality of Life and Mental Health of Women with Diabetes Type 2
}

\author{
Tavakoli Khormizi S.A.R. ${ }^{1}$ MSc, Hamedinia M. ${ }^{2}$ PhD, Amiri Parsa T. ${ }^{3} M S c$ \\ Khadmosharie M. ${ }^{4}$ MSc, Azarnive M.S.* MSc
}

\begin{abstract}
*Exercise Physiology Department, Literature \& Humanities Sciences Faculty, University of Zabol, Zabol, Iran ${ }^{1}$ Exercise Physiology Department, Literature \& Humanities Sciences Faculty, University of Zabol, Zabol, Iran 2Exercise Physiology Department, Physical Education \& Sport Sciences Faculty, Hakim Sabzevari University, Sabzevar, Iran

${ }^{3}$ Exercise Physiology Department, Physical Education \& Sport Sciences Faculty, Ferdowsi University of Mashhad, Mashhad, Iran

${ }^{4}$ Exercise Physiology Department, Physical Education \& Sport Sciences Faculty, Razi University of Kermanshah, Kermanshah, Iran
\end{abstract}

\begin{abstract}
Aims: Type 2 diabetes is a metabolic disorder that affects the quality of life and mental health of the patients. There are different recommendations about doing effective exercise for diabetic patients. The aim of this study was to evaluate the effect of two aerobic trainings and a detraining periods on quality of life and mental health in women with type 2 diabetes.

Materials \& Methods: In this Semi-experimental research in 2011 in Sabzevar City, Iran, 33 women with type 2 diabetes in 40-60 years age range were selected purposefully and were divided into 3 groups each contain 11 samples; control, daily 5-week aerobic exercise and every other day 10-week aerobic exercise. Aerobic training was conduct with an intensity of $60-70 \%$ of maximum heart rate for $30-60 \mathrm{~min}$ per session. The research instruments were the Quality of Life and Mental Health questionnaires. Data analyses were done in SPSS 18 software by repeated measures ANOVA, one-way ANOVA and Tukey post hoc tests.

Findings: There were significant differences between quality of life $(\mathrm{p}=0.002)$ and mental health $(\mathrm{p}=0.04)$ of training groups and control group, but two training groups were not significantly different. There were also significant differences in physical $(p=0.004)$ and psychosocial $(p=0.004)$ domains of quality of life between the three groups at 5- and 10-weeks after the start of the exercises.

Conclusion: Daily 5-week and every other day 10-week aerobic exercises improves mental health and quality of life in women with type 2 diabetes, but the benefits are not maintained after 2 months of detraining.
\end{abstract}

\section{Keywords}

Diabetes Mellitus Type 2 [http://www.ncbi.nlm.nih.gov/mesh/68003924];

Exercise [http://www.ncbi.nlm.nih.gov/mesh/68015444];

Quality of Life [http://www.ncbi.nlm.nih.gov/mesh/68011788];

Mental Health [http://www.ncbi.nlm.nih.gov/mesh/68008603]

\footnotetext{
* Corresponding Author

Tel: +9854312322030

Fax: +9854312322030

Address: Exercise Physiology Department, Literature \& Humanities Sciences Faculty, University of Zabol, Shahid

Mofateh Street, Jahad Keshavarzi Square, Zabol, Iran. Postal Code: 9861673831

azarnive.m@gmail.com

Received: November 4, $2014 \quad$ Accepted: May 25, 2015 ePublished: September 20, 2015
} 


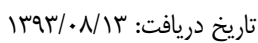

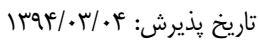

azarnive.m@gmail.com :نويسنده مسئول: تاريخ:

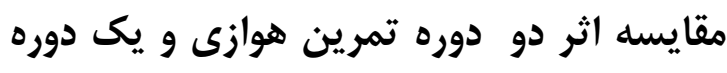
بى متمرينى بر كيفيت زندكَى و سلامت روان زنان

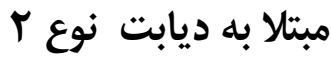

\section{مقدمه}

ديابت از جمله بيمارىهاى متابوليك است كه مشخصه آن افزايش

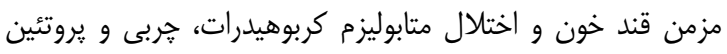
است. اين بيمارى در نتيجه وجود نقايصى در ترشح انسولين، كاركرد انسولين يا هر دو ايجاد مىشود [1]]. اين بيمارى شايع و يرهزينه

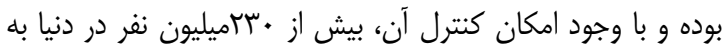

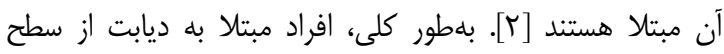

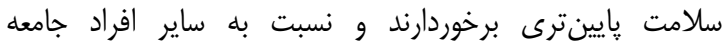
هزينههاى بيشترى را براى درمان مصرف مى كنند [ب]. افزايش بردين

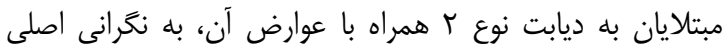

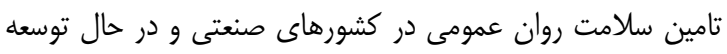

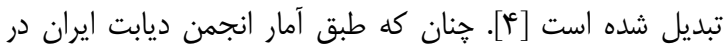

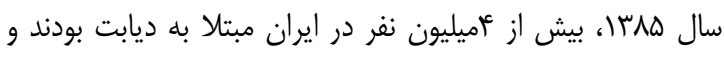

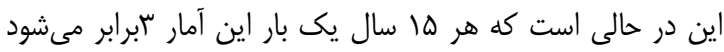

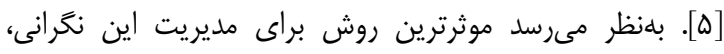

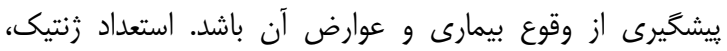

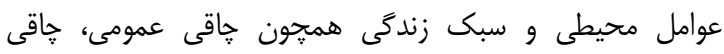

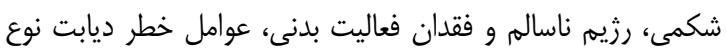

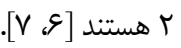

بيمارى ديابت، كيفيت زندگى بيماران را تقريباً در تمام ابعاد زندگى مئى كاهش مىدهد كه طبق تعريف سازمان جهانى بهداشت شامل

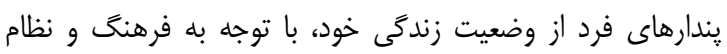

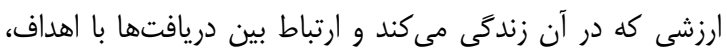

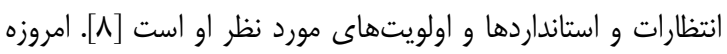

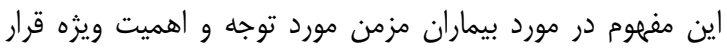

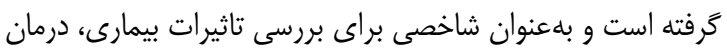

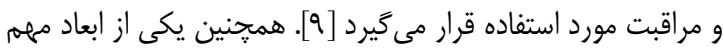
سلامتى، سلامت روان است. بنا به تعريفى كه كارشناسان سازمان جهانى بهداشت مطرح مى كنند، سلامت ذهن عبارت از قابليت

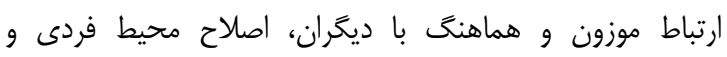
اجتماعى، حل تضادها و تمايلات شخصى بلطور منطقى، عادلانه و

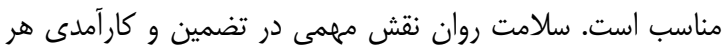
جامعهاى ايفا مى كند [ · [1].

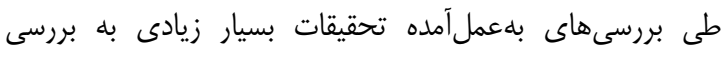

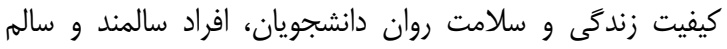
يرداختهاند و تعدادى نيز به بررسى اين فاكتورها در بيماران ديابتى سئى

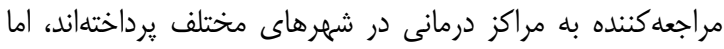

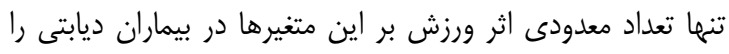

MSc سيدعليرضا توكلى خورميزى

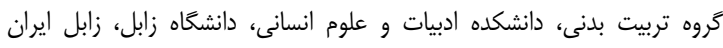

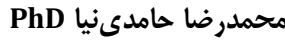

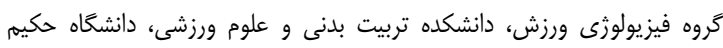

سبزوارى، سبزوار، ايران

طيبه اميرى ويارسا MSc

كروه فيزيولوزى ورزش، دانشكده تربيت إيبا بدنى و علوم ورزشى، دانشخاه فردوسى مشهد، مشهد، ايران

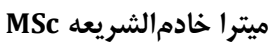

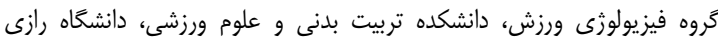
كرمانشاه، كرمانشاه، ايران

MSc مرضيهالسادات آذرنيوه

كروه تربيت بدنى، دانشكده ادبيات و علوم انسانى، دانشخاه زابل، زابل ايران

جكيده

الهداف: ديابت نوع r اختلالى متابوليسمى است كه كيفيت زندكى و

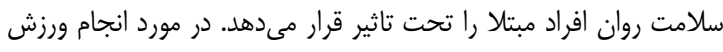
موثر براى اين بيماران توصيههاى مختلفى وجود دارد. هدف مطان مطالعاد

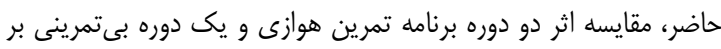

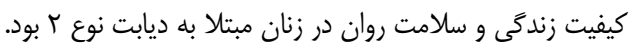

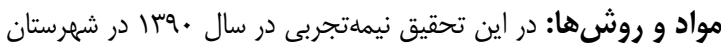

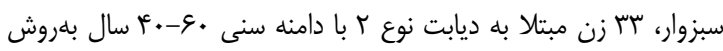

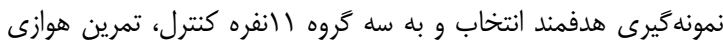

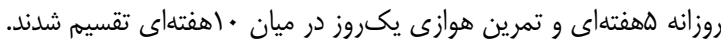

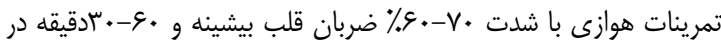

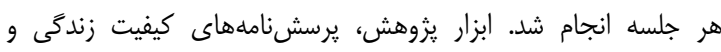

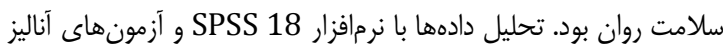
واريانس با اندازهيرى مكرر و يكىطرفه و آزمون تعقيبى توكى انجام

يذيرفت.

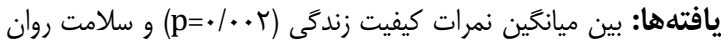

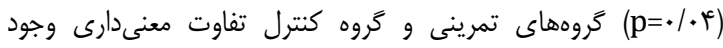

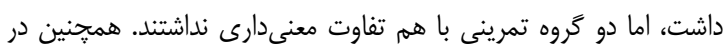

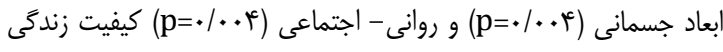

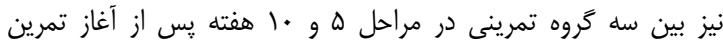
تفاوت معنى دارى مشاهده شد.

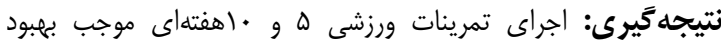

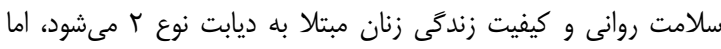

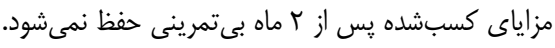

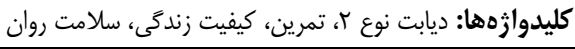

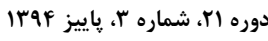

فصلنامه افق دانش 


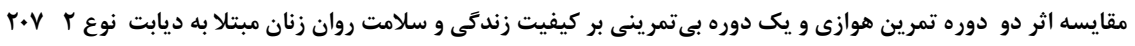

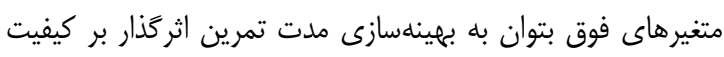

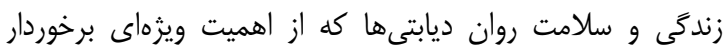

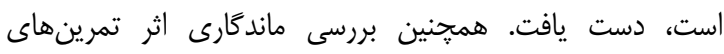

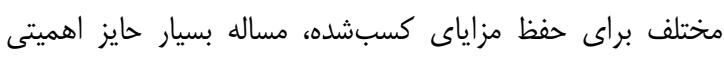

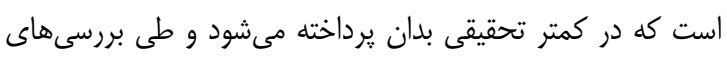

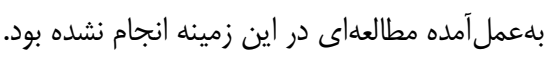

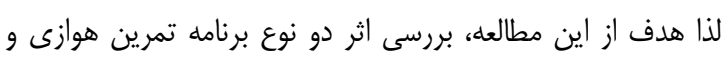

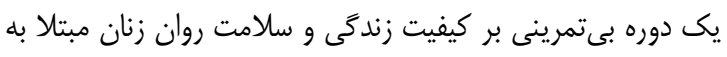

$$
\text { ديابت نوع ب بود. }
$$

\section{مواد و روشها}

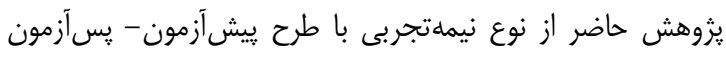

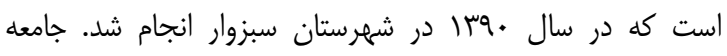

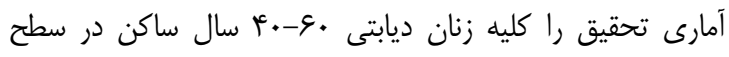

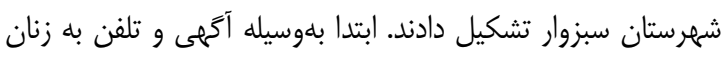

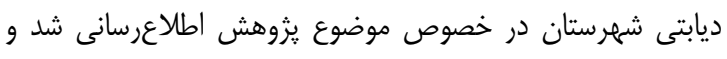

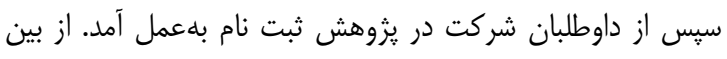

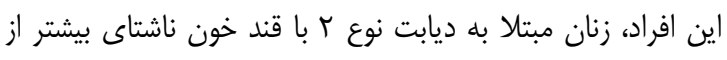

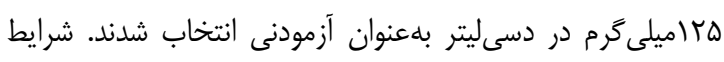

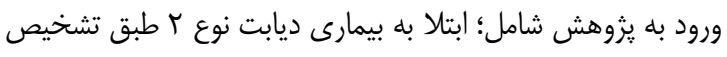

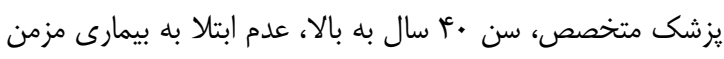

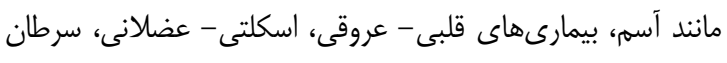

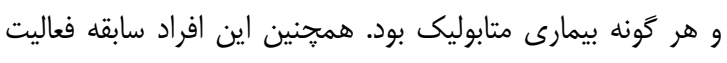

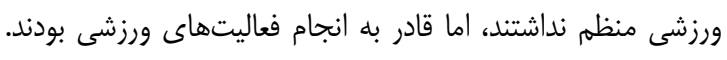

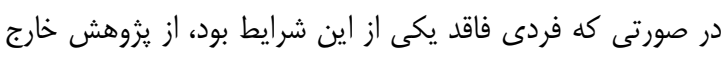

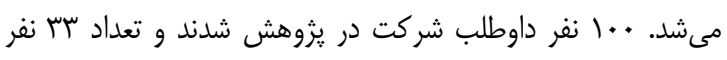

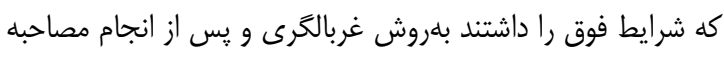

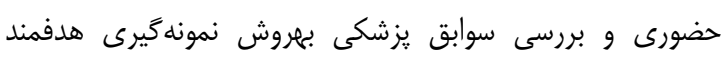

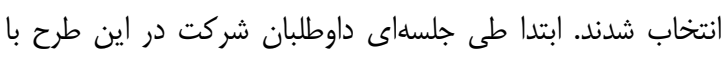

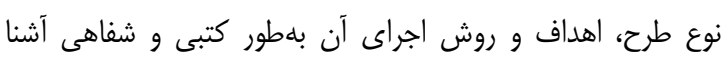

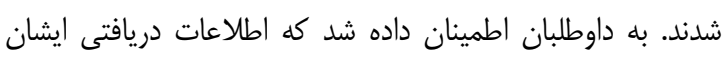

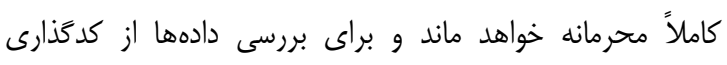

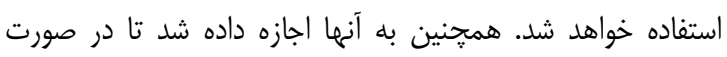

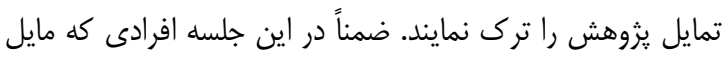

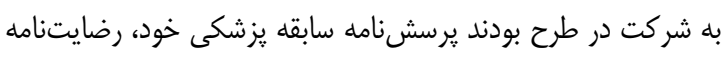

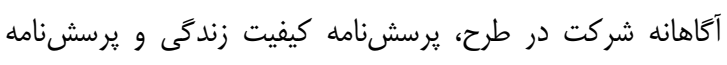

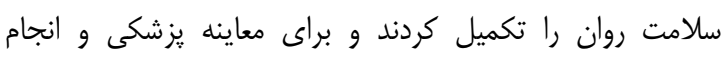

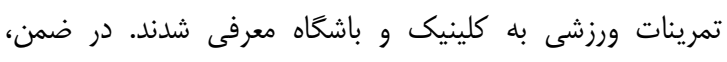

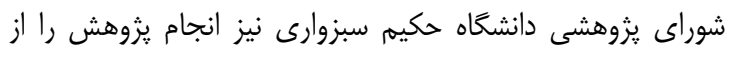

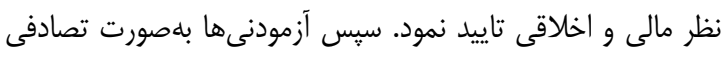

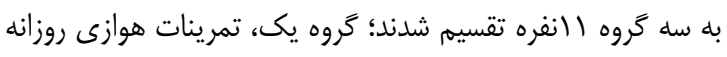

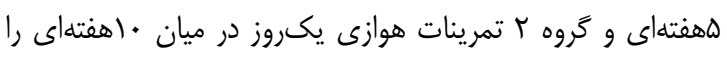

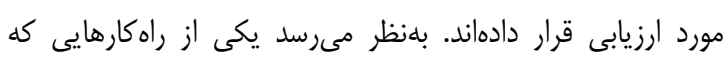

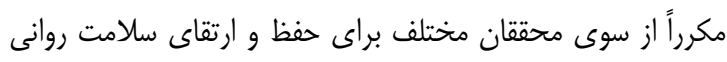

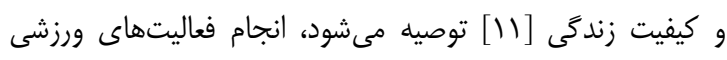

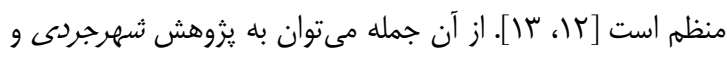

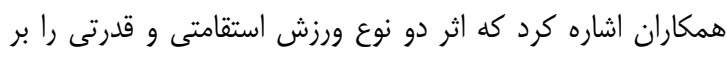

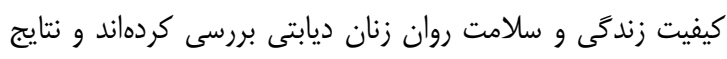

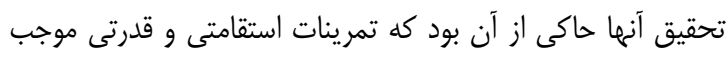

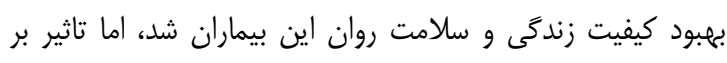

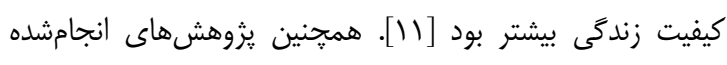

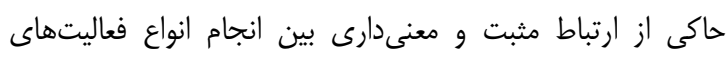

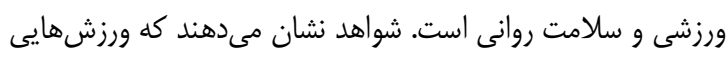

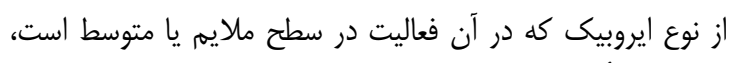

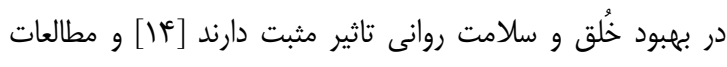

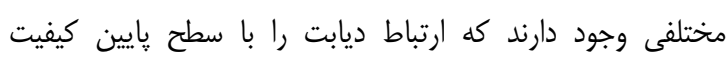

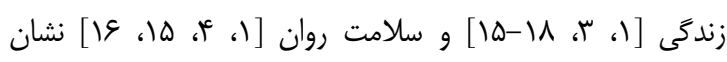

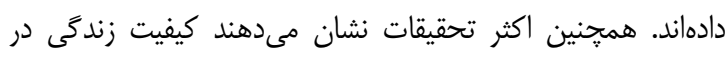

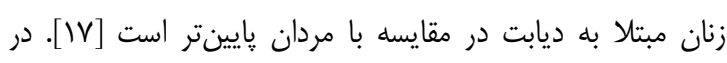

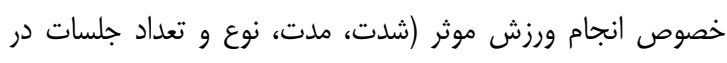

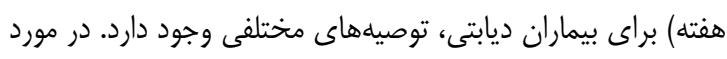

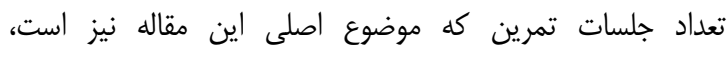

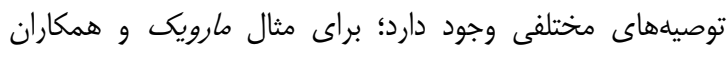

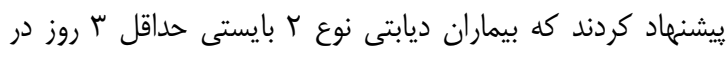

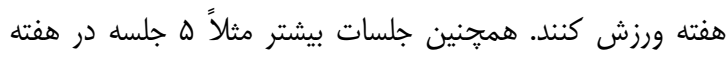

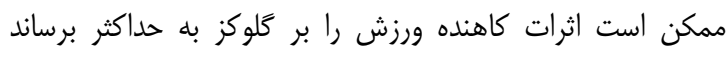

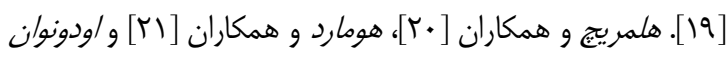

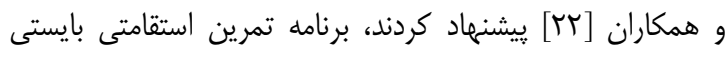

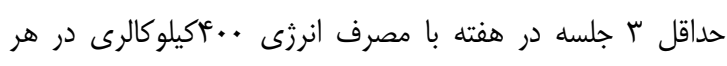

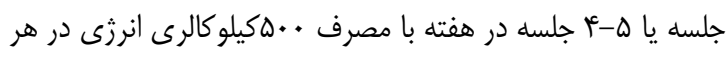

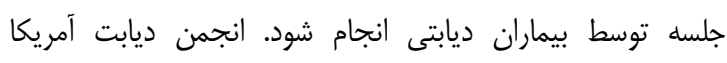

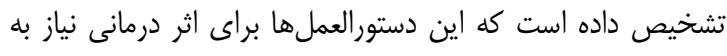

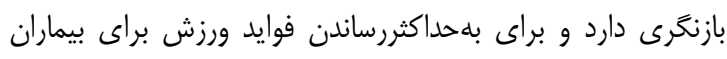

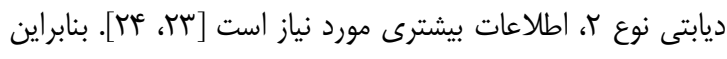

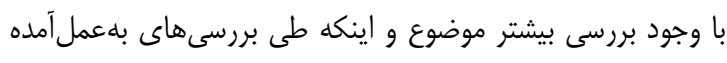

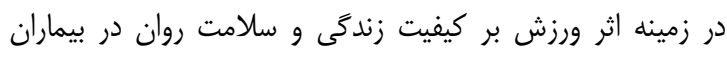

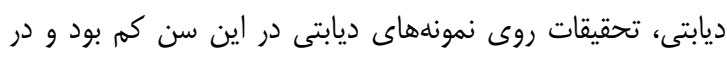

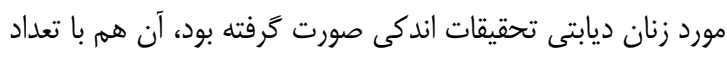

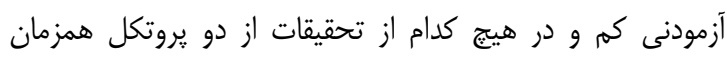

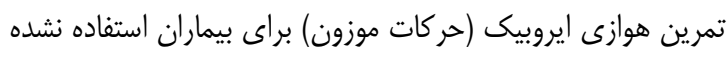

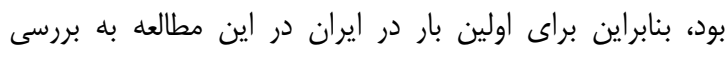

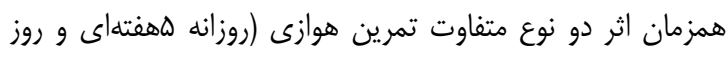

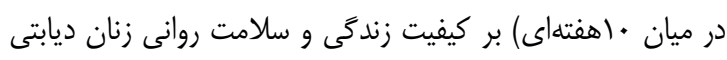

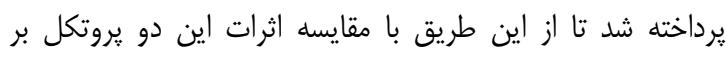


اطلاعات جمعيتشناختى (سن، جنس، تحصيلات، شغل) توسط

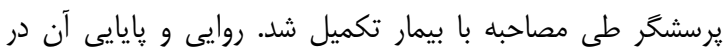

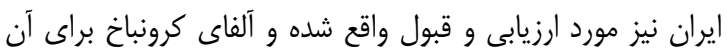

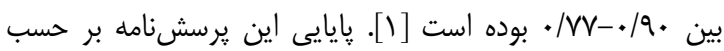

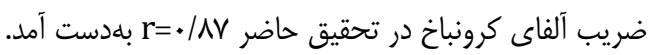

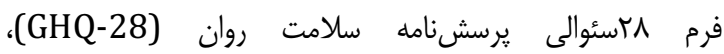

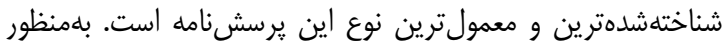

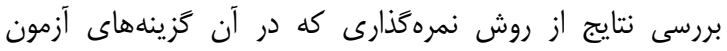

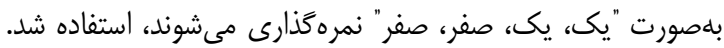

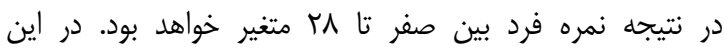

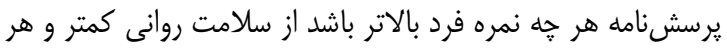

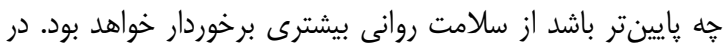
نتايج بررسى كلدبرك و مارى نشان داده است كه متوسط حساسيت

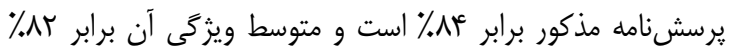

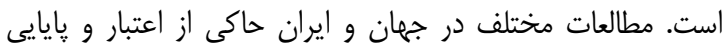
بالاى يرسشنامه GHQ-28 است، براى مثال سعادتمند و نوربالا،

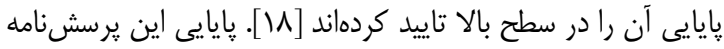

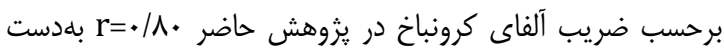

يس إز استفاده از آزمون آمارى كولموكروف- اسميرنوف براى

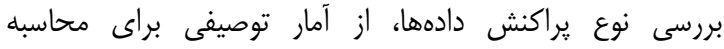

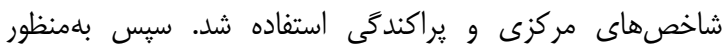

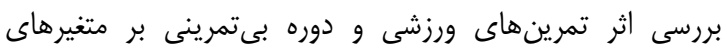

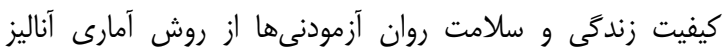

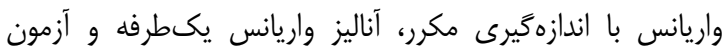

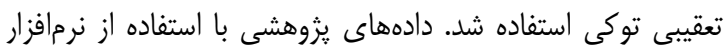
SPSS 18

جدول () مقايسه اطلاعات آمار توصيفى (ميانكين آمارى) مربوط به كروههاى

\begin{tabular}{|c|c|c|c|}
\hline معنىدارى سطح & كنترل & كَروه تمرين روز & 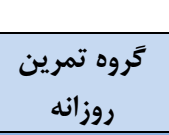 \\
\hline$\cdot / V T$ & $\Delta F / 1 \cdot \pm V / . \varphi$ & $\Delta T / V \cdot \pm r / \Lambda$. & $\begin{array}{l}\text { سن (سال) } \\
\Delta r / r \cdot \pm r / v .\end{array}$ \\
\hline \multicolumn{3}{|c|}{ طول دوره بيمارى (سال) } & $\begin{array}{c}\text { طول دوره بيمارى } \\
\text { V/VVIr/q. }\end{array}$ \\
\hline Tr/ & $V T / F \Lambda \pm I \cdot / E T$ & $89 / 8) \pm 11 / \Delta \mathrm{V}$ & $\begin{array}{l}\text { وزن (كيلوكَرم) } \\
99 / V 9 \pm 9 / 9 \Lambda\end{array}$ \\
\hline$\cdot / \Delta r$ & $r q / \Lambda r \pm r / \Delta V$ & 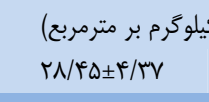 & 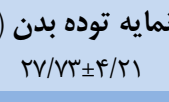 \\
\hline \multicolumn{4}{|c|}{ مصرف روزانه متفورمين (قرص در روز) } \\
\hline \multicolumn{4}{|c|}{ مصرف روزانه كَلىبنَكلامين (قرص در روز) } \\
\hline
\end{tabular}

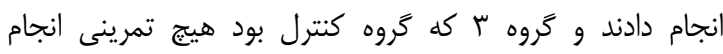
نمىدادند. كروه يك هر روز بلهز

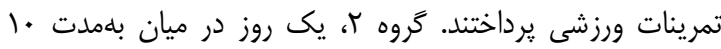

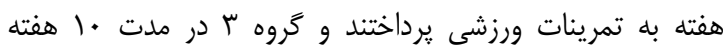
يروتكل يزوهش به فعاليتهاى عادى برداختند و تمرينات ورزشى برى

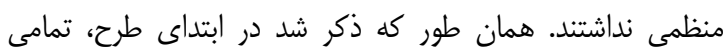

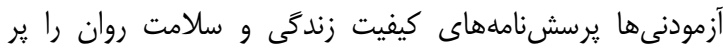

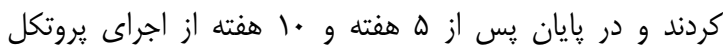

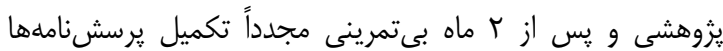
براى همه آزمودنى ها (سه كَروه) تكرار شد.

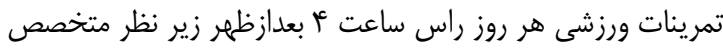

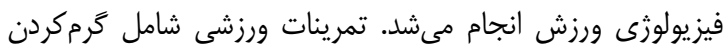

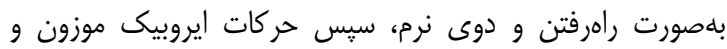

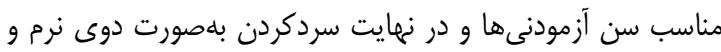

$$
\text { آهسته و حركات كششى انجام مى شد. }
$$

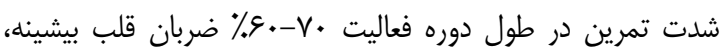

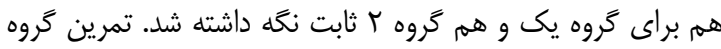

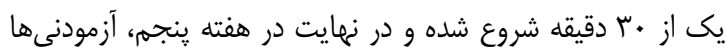
به زمان •و دقيقه تمرين رسيدند.

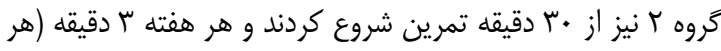

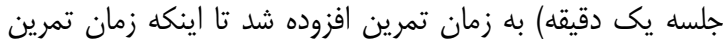

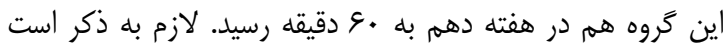

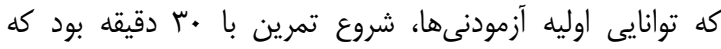

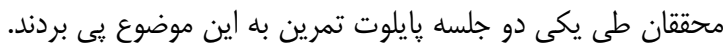

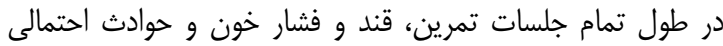
ديخر توسط يرستار كنترل شد.

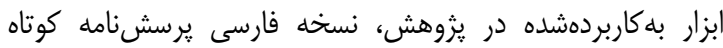

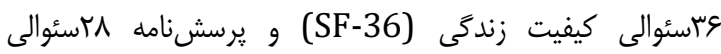
سلامت روان SFHQ-28) بود. برسشنامه SF-36 بهمنظور

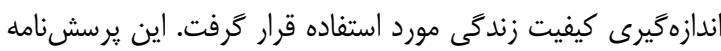

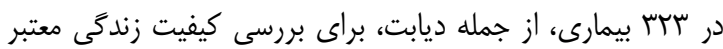

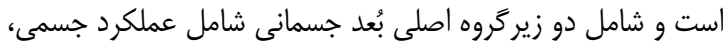

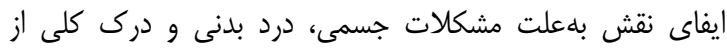

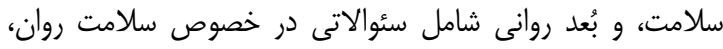

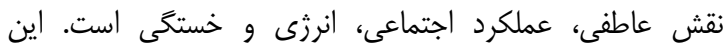

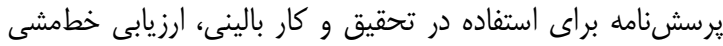

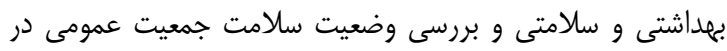

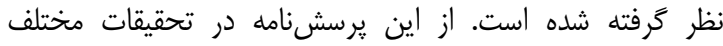

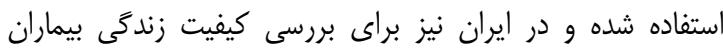

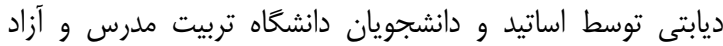

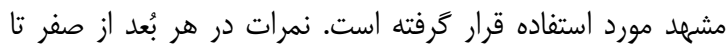
.. اتعيين مى شود. در ابتداى برسشنامه نيز يك سئوال در مورد 


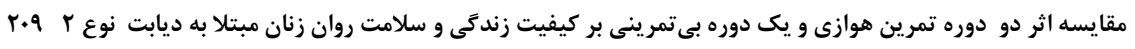

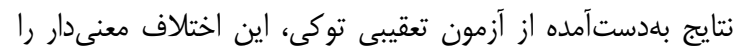

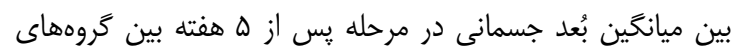

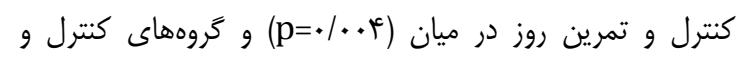

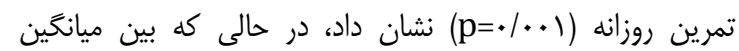

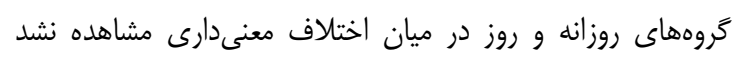
(p=×|VY)

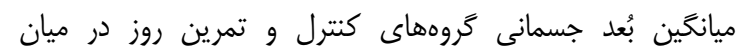
(p=•/1)

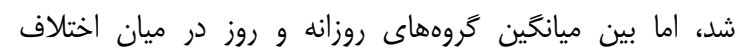

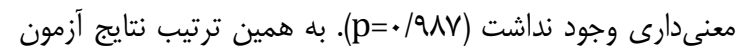

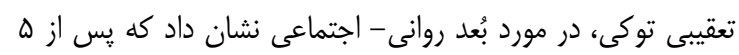

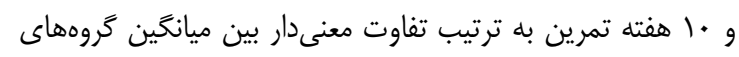

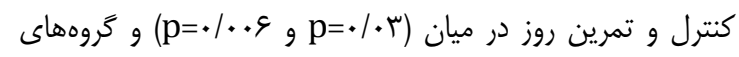

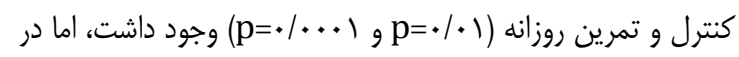

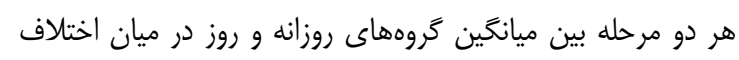

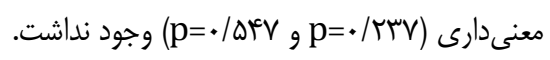
متغير سلامت روان بين سه گروه تمرينى تفاوت معنىدارى داشت

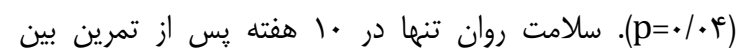
ميانخين گروهها تفاوت معنىدار داشت، اما در ه هفته يس إز از تمرين

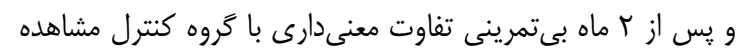

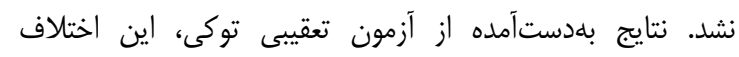

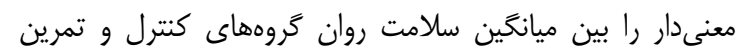

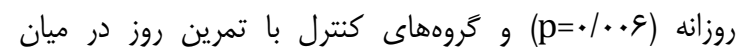

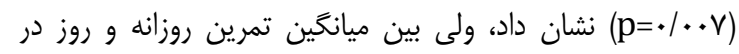

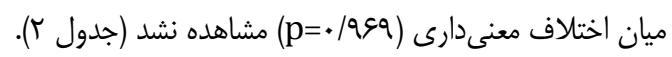

متغيرهاى سن، قد، وزن، نمايه توده بدن، طول دوره بيمارى و

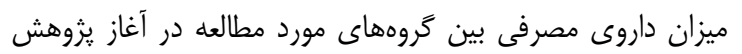

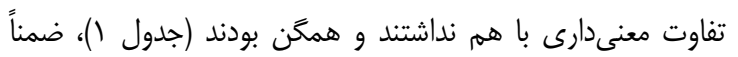

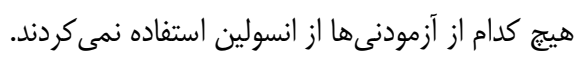

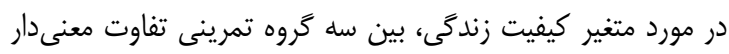

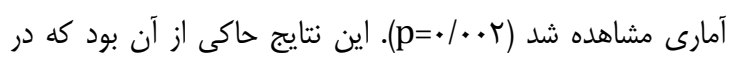

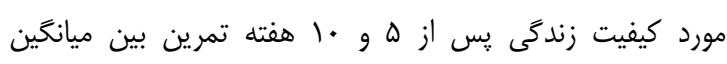

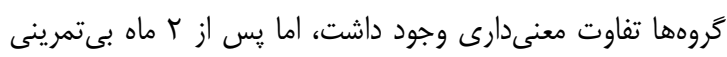

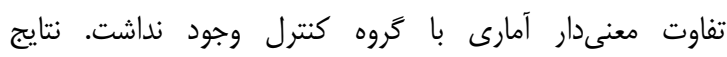

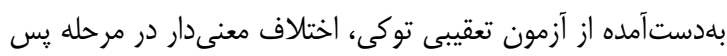

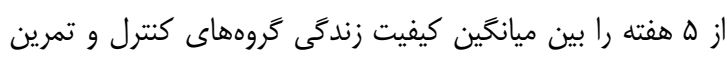

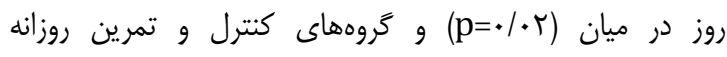

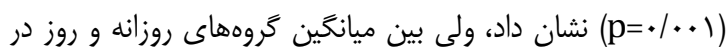

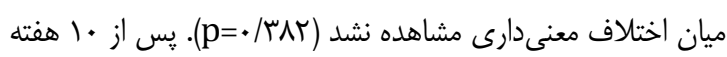

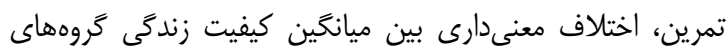

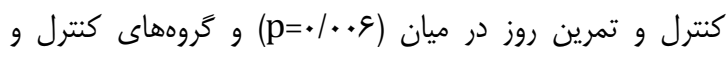

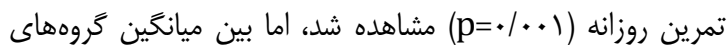

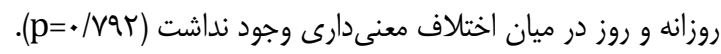

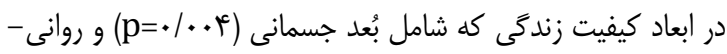

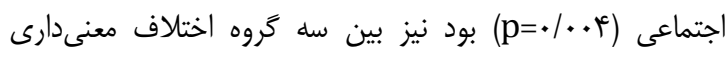

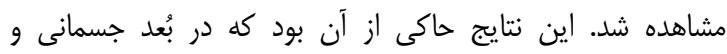

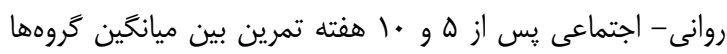

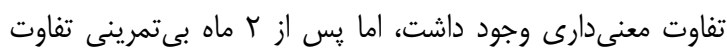

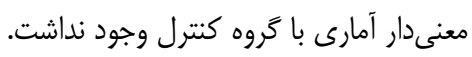

جدول r) نتايج آزمون آمارى مربوط به مقايسه ميانگَين نمرات كيفيت زندگى و سلامت روان بين كروهها

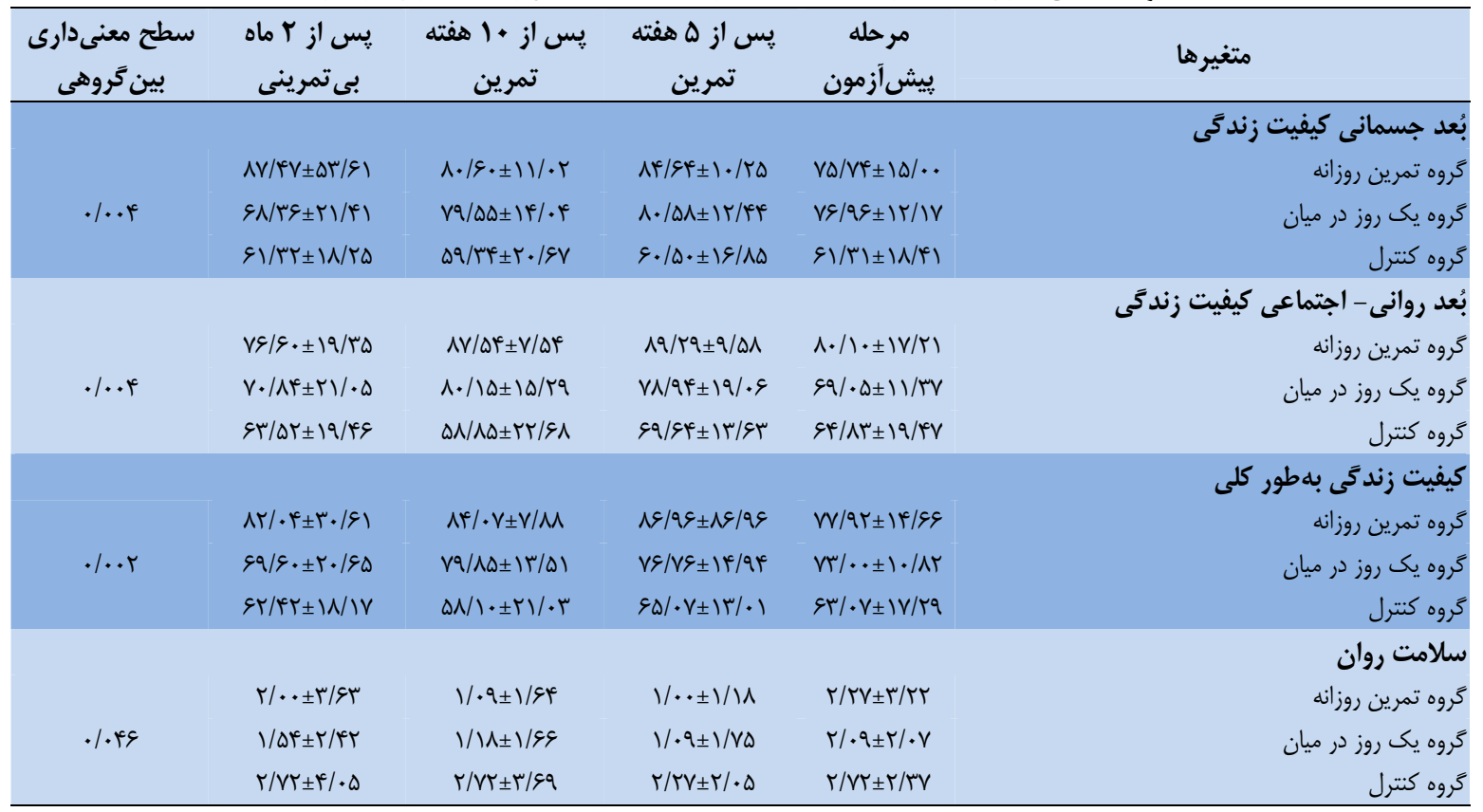


آنها حاكى از آن بود كه تمرينات ورزشى اثرات مثبت بيشترى روى

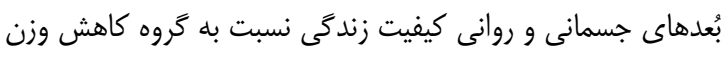

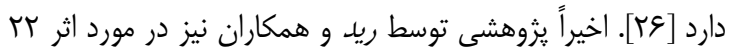

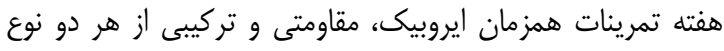

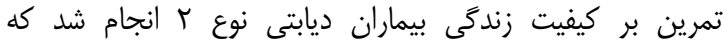

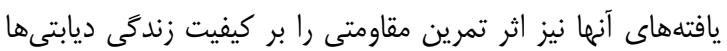

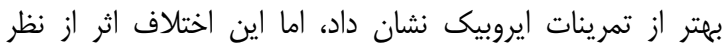

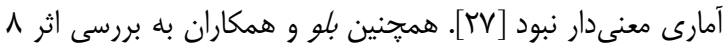

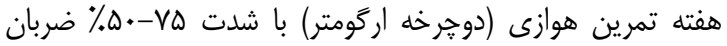

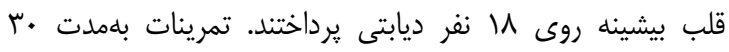

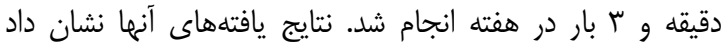
تمرين ورزشى موجب بجبود غيرمعنى دار كيفيت بيماران ديابتى شد إندان

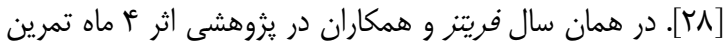

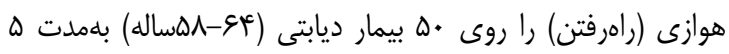

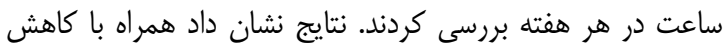

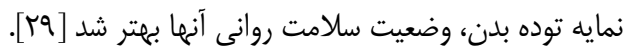
به كزارش وزارت بهداشت، درمان و خدمات انسانى ايالات متحده،

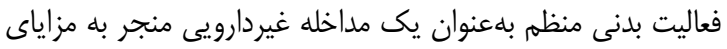

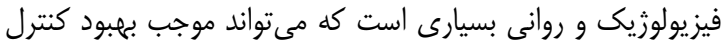

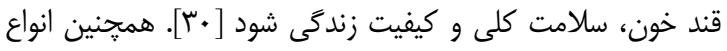
مختلف ورزش (هوازى، مقاومتى يا نوع تركيبى) بهعنوان يكى ابزار

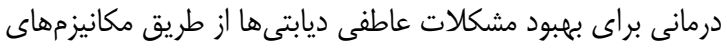

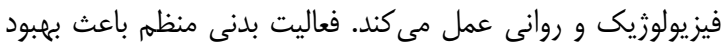

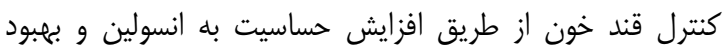

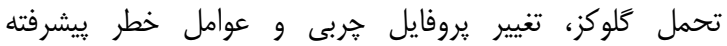

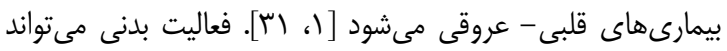

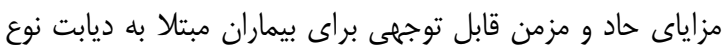

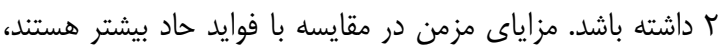

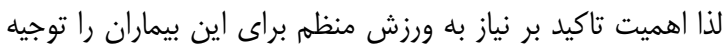

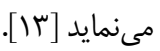

همجنين طى بررسى مطالعات مربوطه دريافتيم كه در مورد اثر

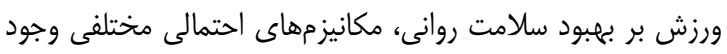

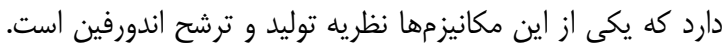

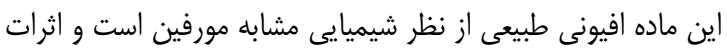

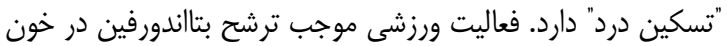

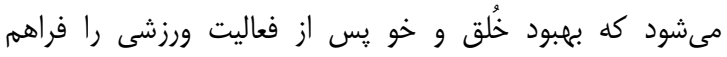

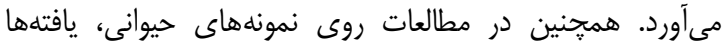

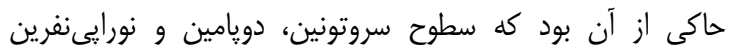

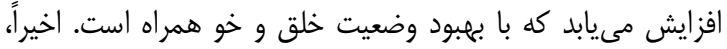
افزايش فعاليت لوبهاى مغز و هيبوكامٍ موجب افز افزايش سطوح

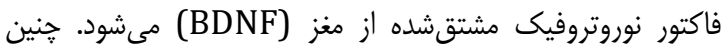

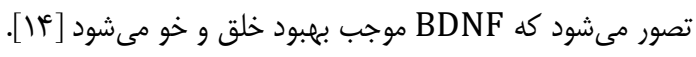

نتايج يزوهش حاضر نشان داد كه تمرين ههفتهاى روزانه و

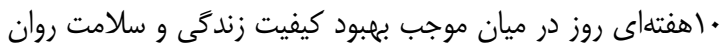

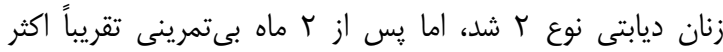

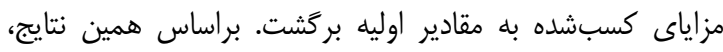

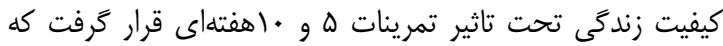

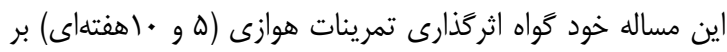

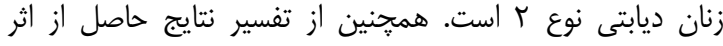

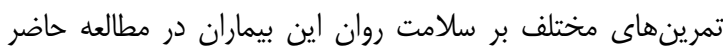

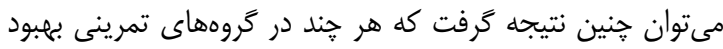

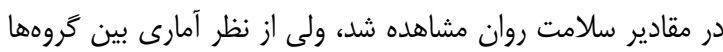

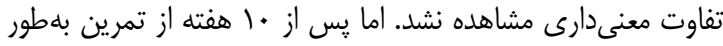

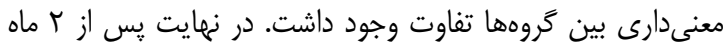

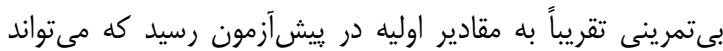

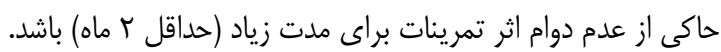

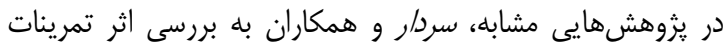

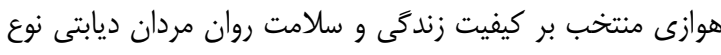

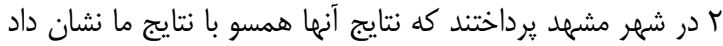

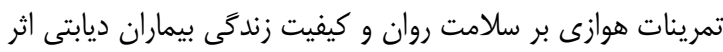

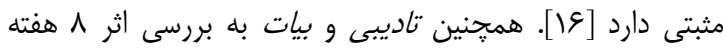

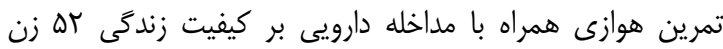

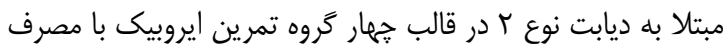

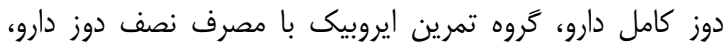

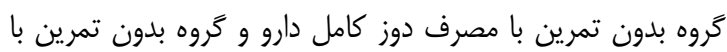

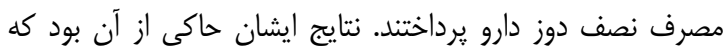

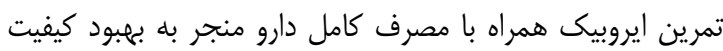

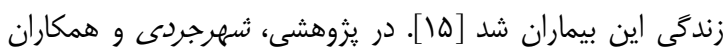

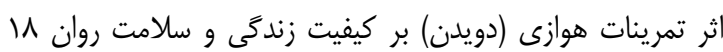

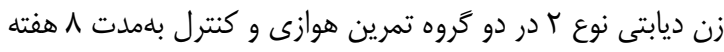

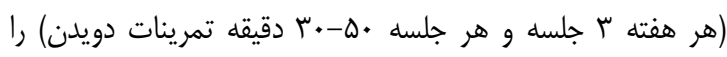
مورد بررسى قرار دادند. نتايج اين يثوهش نيز همسو با تحقيق ندات

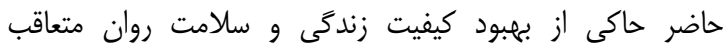

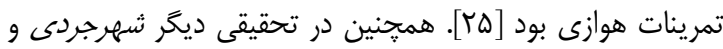

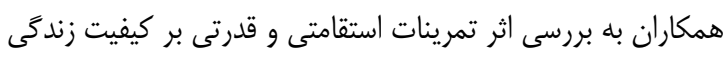

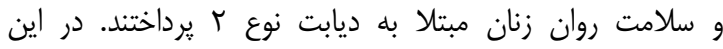

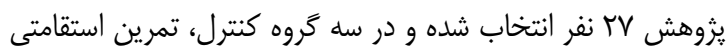

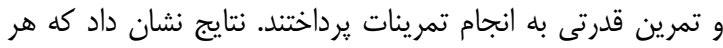

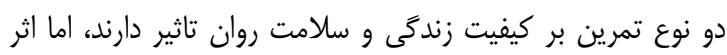

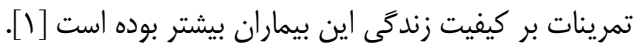

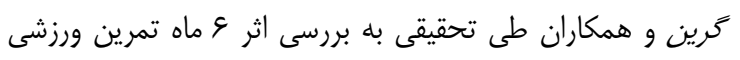

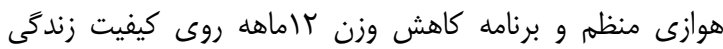
فال

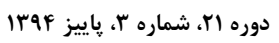

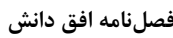




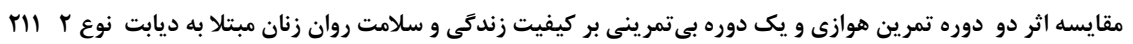

\section{منابع}

1- Shahrjerdi Sh, Shavandi N, Hosseini Sheikhi R, Shahrjerdi Sh. Effect of strength and endurance training on metabolic factors, quality of life and mental health in women with type 2 diabetes. J Shahrekord Uni Med Sci. 2010;12(3):85-93.

2- Keykha M, Janghorbani M, Amini M. The prevalence of type 2 diabetes, metabolic syndrome and cardiovascular risk factors Pyshdyabt and their first-degree relatives of patients with type 2 diabetes. J Kerman Uni Med Sci. 2013;20(2):115-28. [Persian]

3- Gram B, Christensen R, Christiansen C, Gram J. Effects of nordic walking and exercise in type 2 diabetes mellitus: A randomized controlled trial. Clin J Sport Med. 2010;20(5):355-61.

4- Lincoln AK, Shepherd A, Johnson PL, CastanedaSceppa C. The impact of resistance exercise training on the mental health of older Puerto Rican adults with type 2 diabetes. J Gerontol Series B Psychol Sci Soc Sci. 2011;66(5):567-70.

5- Haririan HR, Moghadasian S, Aghajanlo A. Quality of life in diabetic patients attending the Diabetes Center, Tabriz University of Medical Sciences, in 1386. Iran J Diabetes Lipid Disord. 2010;9(2):152-60. [Persian]

6- Fakhrzadeh H, Ghaderpanahi M, Sharifi F, Badamchizadeh Z, Mirarefin M, Pour Ebrahim R, et al. The relationship between physical activity and risk of type 2 diabetes among 64-24 year old residents of district 17 of Tehran. J Diabetes Metabolism. 2011;10(2):170-9. [Persian]

7- Colberg SR, Sigal RJ, Fernhall B, Regensteiner JG, Blissmer BJ, Rubin RR, et al. Exercise and Type 2 Diabetes. Diabetes care. 2010;33(12):2692-6.

8- Ahmadi A, Hasanzade J, Rahimi Medise M, Lashkari L. Factors influencing quality of life in patients with type 2 diabetes and Bakhtiari province. North Khorasan Uni J Med Sci. 2012;3(1):7-13. [Persian]

9- Hadipour M, Abolhasani F, Molavi Vardanjani H, Eybpoosh S. Individual and environmental determinants of health related quality of life in Iranian patients with type II diabetes. Iran South Med J. 2014;16(6):428-35. [Persian]

10- Solgi Z, Saeedipoor B, Abdolmaleki P. Study of psychological well-being of physical education students of Razi university of Kermanshah. J Kermanshah Uni Med Sci. 2010;13(2):172-8.

11- Shahrjerdi Sh, Shavandi N, Golpaigani M, Sheikh Hosseini R. Impact strength and resistance training on glycemic control, quality of life and mental health in women with type 2 diabetes. Iran J Diabetes Metab. 2010;9(1):35-44. [Persian]

12- Morgan AJ, Parker AG, Alvarez-Jimenez M, Jorm AF. Exercise and Mental Health: An Exercise and Sports Science Australia Commissioned Review. J Exerc Physiol Online. 2013;16(4).122-35.

13- Yavari A, Najafipur F, Asgarzade A, Niafar M, Mobseri M, Dabagh Nikokheslat S. Effects of aerobic training, strength and composition on glycemic control and cardiovascular risk factors in type 2 diabetic patients. Med J Tabriz Uni Med Sci. 2012;33(4):82-91. [Persian]

14- Koehl M, Meerlo P, Gonzales D, Rontal A, Turek FW, Abrous DN. Exercise-induced promotion of hippocampal cell proliferation requires beta-endorphin. FASEB J. 2008;22(7):2253-62.

15- Tadibi V, Bayat Z. Effect of eight weeks of aerobic exercise and pharmacological intervention on quality of
از طرف ديخر، ورزش باعث اثرگذارى و بهبود بسيارى از عوامل خطرزاى شناختهشده بيمارىهاى قلبى - عروقى با اثرات مثبتى كه ونه

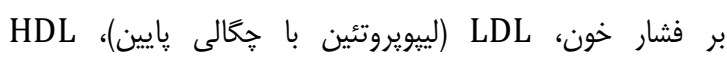

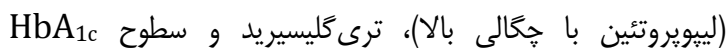

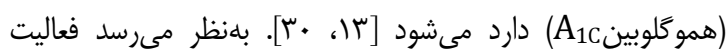

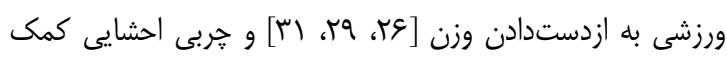

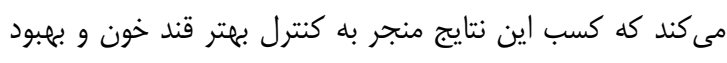

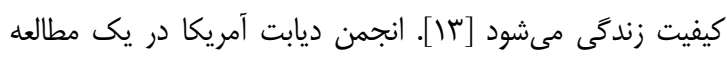

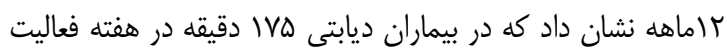

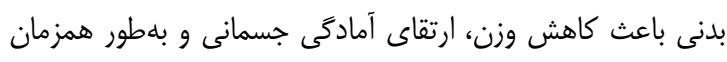

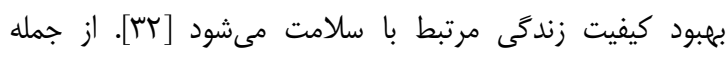

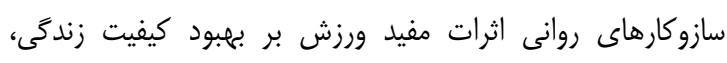

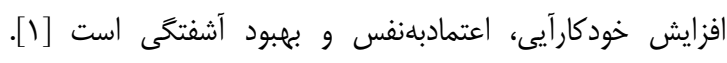

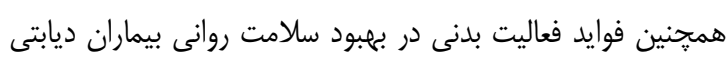

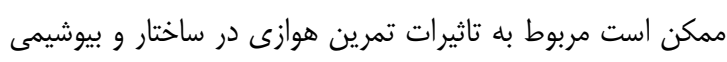

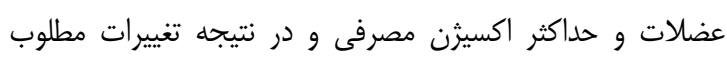
ايجادشده باشد (مثل افزايش آنزيم دى اكسيداتيو و افزايش دانسيرن دانسيته دئه

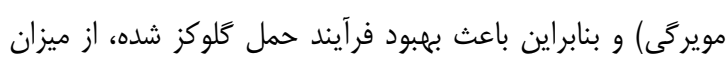

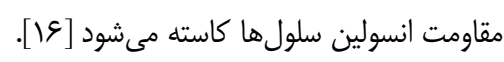
از محدوديتهاى يزوهش حاضر، استفاده از نمونه گيرى دردسترس

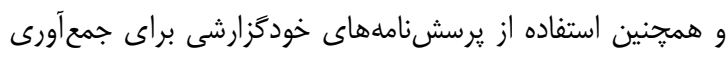

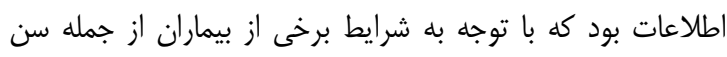

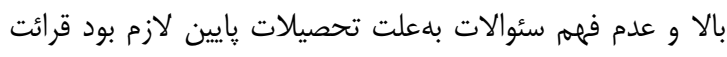

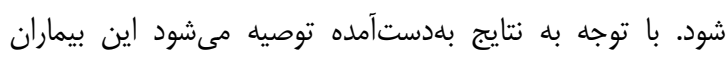

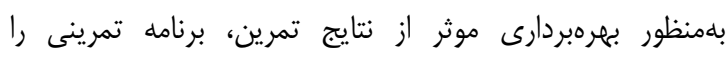

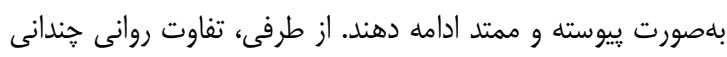
بين دو گروه تمرينى وجود نداشت كه اين مساله مىتواند مبنايى مئى براى طراحى برنامههاى تمرينى با توجه به شرايط مختلف براي ائ اين بيماران باشد.

\section{نتيجه كيرى}

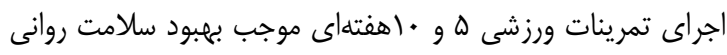

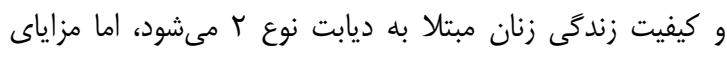

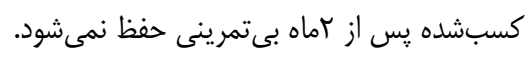

تشكر و قدردانى: در يايان، نويسندكان مراتب سياس خود را از

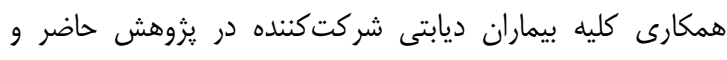

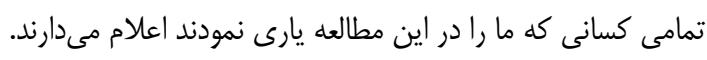
تاييديه اخلاقى: اين يزوهش از نظر اخلاقى در شوراى يثوهشى

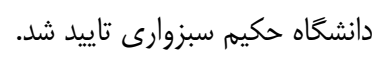
تعارض منافع: موردى توسط نويسندكان گزارش نشده است. منابع مالى: توسط نويسندكان تامين شد. 
care in diabetes-2012. Diabetes Care. 2012;35(1):S11S63.

25- Shahrjerdi Sh, Shavandi N, Sheikh Hosseini R. The effect of aerobic exercise on metabolic factors, quality of life (QOL) and mental health (MH) in women with type II diabetes. J Arak Un Med Sci. 2010;12(4):25-35.

26- Green AJ, Fox KM, Grandy S. Impact of regular exercise and attempted weight loss on quality of life among adults with and without type 2 diabetes mellitus. J Obes. 2011;2011:1-6.

27- Reid R, Tulloch H, Sigal R, Kenny G, Fortier M, McDonnell L, et al. Effects of aerobic exercise, resistance exercise or both, on patient-reported health status and well-being in type 2 diabetes mellitus: a randomised trial. Diabetologia. 2010;53(4):632-40.

28- Bello AI, Owusu-Boakye E, Adegoke BO, Adjei DN. Effects of aerobic exercise on selected physiological parameters and quality of life in patients with type 2 diabetes mellitus. Int J Gen Med. 2011;4:723-27.

29- Fritz T, Caidahl K, Osler M, Östenson C, Zierath J, Wändell P. Effects of Nordic walking on health-related quality of life in overweight individuals with Type 2 diabetes mellitus, impaired or normal glucose tolerance. Diabet Med. 2011;28(11):1362-72.

30- Van der Heijden M, Van Dooren F, Pop VJ, Pouwer F. Effects of exercise training on quality of life, symptoms of depression, symptoms of anxiety and emotional wellbeing in type 2 diabetes mellitus: A systematic review. Diabetologia. 2013;56(6):1210-25.

31- Hayes C, Herbert M, Marrero D, Martins C, Muchnick S. Diabetes and exercise. Diabetes Educ. 2008;34(1):3740.

32- Saremi A. Exercise and type 2 diabetes mellitus: A review of the evidence. Cell Tissue J. 2012;2(3):171-81. [Persian]

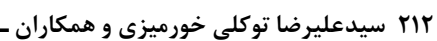

life in women with type 2 diabetes. J Gorgan Uni Med Sci. 2012;14(2):29-35.

16- Sardar MA, Sohrabi M, Shamsian AA, Aminzade R. Effects of aerobic exercise training on the mental and physical health and social functioning of patients with type 2 diabetes mellitus. Iran J Endocrinol Metab. 2010;11(3):251-6. [Persian]

17- Vares Z, Zandi M, Baghaei P, Masudi Alavi N, Mirbagher Ajorpaz N. Quality of life and related factors in diabetic patients referred to a diabetes center in Kashan. J Nurs Res. 2011;5(17):14-22. [Persian]

18- Norbala AA, Bagheri Yazdi A, Mohammad K. The validation of general health questionnaire- 28 as a psychiatric screening too. Hakim Res J. 2009;11(4):4753. [Persian]

19- Marwick, TH, Hordern MD, Miller T, Chyun DA, Bertoni AG, Blumenthal RS, et al. Exercise training for type 2 diabetes mellitus: Impact on cardiovascular risk: a scientific statement from the American Heart Association. Circulation. 2009;119(25):3244-62.

20- Helmrich, SP, Ragland DR, Leung RW, Paffenbarger RS Jr. Physical activity and reduced occurrence of noninsulin-dependent diabetes mellitus. $\mathrm{N}$ Engl J Med. 1991;325(3):147-52.

21- Houmard, JA, Tanner CJ, Slentz CA, Duscha BD, McCartney JS, Kraus WE. Effect of the volume and intensity of exercise training on insulin sensitivity. J Appl Physiol. 2004;96(1):101-6.

22- O’Donovan G, Kearney EM, Nevill AM, Woolf-May K, Bird SR. The effects of 24 weeks of moderate-or highintensity exercise on insulin resistance. Eur J Appl Physiol. 2005;95(5-6):522-8.

23- American Diabetes Association. Standards of medical care in diabetes-2007. Diabetes Care. 2007;30(1):S4-41.

24- American Diabetes Association. Standards of medical 